\title{
PTSD, social anxiety disorder, and trauma: An examination of the influence of trauma type on comorbidity using a nationally representative sample
}

\author{
Katherine A. McMillan*, Gordon J.G. Asmundson \\ Anxiety and Illness Behaviours Laboratory, Department of Psychology, University of Regina, Regina, SK, Canada
}

\section{A R T I C L E I N F O}

\section{Keywords:}

Posttraumatic stress disorder

Social anxiety disorder

Trauma

Traumatic events

Comorbidity

Anxiety

\begin{abstract}
A B S T R A C T
Posttraumatic stress disorder (PTSD) and social anxiety disorder (SAD) are highly comorbid (Collimore et al., 2010). Trauma may present a shared environmental factor contributing to the development of comorbidity; however, existent research has been hampered by use of restrictive samples and limitations in the range of traumas investigated. The current study examines the relationship between a broad range of potentially traumatic events and the comorbidity between PTSD and SAD using Wave 2 of the National Epidemiological Survey of Alcohol and Related Conditions $(n=34,653)$. Multiple logistic regressions and cross-tabulations were used to evaluate differences in the prevalence of potentially traumatic events among those who met criteria for comorbid PTSD-SAD compared to those with PTSD without SAD and SAD without PTSD. Those in the comorbid PTSD-SAD group were significantly more likely than those in the PTSD without SAD or SAD without PTSD groups to report experiencing specific types of assaultive violence, childhood maltreatment, and other shocking events. Associations between comorbidity and childhood maltreatment were significant for females only. Individuals diagnosed with comorbid PTSD-SAD are more likely than those diagnosed with either disorder alone to report exposure to specific types of traumatic events within their lifetime.
\end{abstract}

\section{Introduction}

Posttraumatic stress disorder (PTSD) and social anxiety disorder (SAD) frequently co-occur. Indeed, rates of DSM-IV diagnosed pastyear SAD among individuals with PTSD range from $14.8 \%$ to $46 \%$ within veteran and civilian samples (Collimore et al., 2010). Preliminary evidence from treatment-seeking populations suggests that comorbid PTSD and SAD is associated with higher levels of depression and anxiety, and lower levels of mental and social functioning, compared to those with either disorder alone (Zayfert et al., 2005). Similarly, a study of veterans documented an increased suicide risk when comorbid PTSD and SAD was present compared to PTSD alone, even after adjusting for PTSD symptom severity (Kashdan et al., 2006). The high rates of comorbidity in conjunction with deleterious mental health outcomes underscores the need for a greater understanding of comorbid presentations of PTSD and SAD.

A number of factors have been proposed to explain the cooccurrence of PTSD and SAD, including genetic, psychological, physiological, and environmental influences (for review see Collimore et al., 2010). Research regarding environmental effects suggests that, like PTSD, trauma exposure may also precipitate SAD onset (Ost, 1987; Ost and Hugdahl, 1981; Stemberger et al., 1995). Much information exists linking trauma characteristics to the development of PTSD, such as intensity or duration (Golding, 1999), interpersonal context (Howgego et al., 2005), and direct versus indirect exposure (Kim et al., 2009; Pietrzak et al., 2011). Much less is known about the relationship between $\mathrm{SAD}$ and trauma exposure; however, research suggests that $44-58 \%$ of individuals with SAD are able to identify a salient event which precipitated symptom onset (Ost, 1987; Ost et el., 1981; Stemberger et al., 1995).

Much of the research examining the relationship between SAD and trauma has been circumscribed to the study of childhood adversity. Individuals with SAD report higher rates of verbal aggression by an adult (Magee, 1999), childhood physical abuse (Acarturk et al., 2009; Chartier et al., 2001), sexual abuse (Acarturk et al., 2009; Bandelow, et al., 2004; Chartier et al., 2001; Magee, 1999), psychological abuse (Acarturk et al., 2009), and emotional abuse (Kuo et al., 2011), with some between-sex differences noted (Chartier et al., 2001). Evidence suggests that the relationship between childhood sexual abuse and SAD may be particularly robust. Magee (1999) investigated the role of multiple traumatic experiences and chronic adversities during childhood on the onset of phobic disorders. After adjusting for sex, age, birth-cohort, previous phobias, and four highly comorbid disorders (major depression, PTSD, drug and alcohol abuse or addiction), rape or

\footnotetext{
* Corresponding author.

E-mail address: Kmcmillan84@gmail.com (K.A. McMillan).
} 
molestation by a relative was significantly associated SAD onset. The author hypothesized that childhood sexual abuse may lead to selfblame and blame by others, thereby predisposing fear of criticism and increasing risk for the later SAD development.

Within a sample of military veterans who met criteria for PTSD, Orsillo et al. (1996) found that personal shame regarding military service accounted for $3 \%$ of the unique variance in social interaction anxiety. Findings remained significant even after accounting for premilitary social anxiety, combat exposure severity, and difficulty with repatriating, partially supporting the idea that guilt and shame may contribute to PTSD and SAD comorbidity. Zayfert et al. (2005) found increased trauma-related guilt among treatment-seeking individuals with comorbid PTSD and SAD versus those with PTSD alone. In addition, Zayfert investigated the role of shame by examining rates of childhood sexual and physical abuse (traumas known to have a significant association with feelings of shame) and observed higher rates of childhood abuse among individuals with comorbid PTSD and SAD compared to those with PTSD alone. Collimore et al. (2009), on the other hand, failed to demonstrate that assaultive violence had a stronger association with social fears than traumas of a non-assaultive nature; however, findings may have been limited by differences in group sample sizes (i.e., nonassaultive trauma group, $n=20$; assaultive trauma group, $n=128$ ) and use of social fears measures rather than diagnosed $\mathrm{SAD}$.

The need for further understanding the association between different trauma types and the development of comorbid PTSD and SAD versus PTSD without SAD or SAD without PTSD is warranted, as current research is limited to treatment-seeking and military populations, the results of which may not be generalizable. Additionally, while Zayfert et al. (2005) examined differences in reported rates of childhood physical or sexual abuse among individuals with comorbid PTSD and SAD compared to those with PTSD alone, the relationship between trauma type and comorbidity was not the focus of the study; no differentiation was made between childhood sexual abuse and physical abuse or forms of neglect and other traumas were not examined. It remains to be determined whether particular types of traumatic experiences are more closely associated with comorbid PTSD and SAD compared to PTSD without SAD or SAD without PTSD. The current study is the first to provide a comprehensive evaluation of a diverse range of traumatic life events, including distinct types of childhood abuse and neglect, and their association with comorbid PTSD and SAD within a nationally-representative sample. Furthermore, the current study is the first to examine the potential moderating influence of gender on the associations between trauma exposure and PTSD-SAD comorbidity. Patterns of trauma exposure were examined by comparing prevalence rates among those with comorbid PTSD and SAD against those with SAD or PTSD alone to identify traumas unique to the presence of comorbidity.

Strong support exists for the association between interpersonal trauma and PTSD, with current research supporting similar associations for SAD. As such, it was anticipated that intimate partner violence, sexual assault, childhood physical and sexual abuse, and combat would occur more often among individuals with comorbid PTSD and SAD compared to those with PTSD without SAD or SAD without PTSD. Given the relative strength of the relationship between history of trauma and the presence of PTSD (American Psychiatric Association, 2013), it was anticipated that the majority of the associations would emerge as significant when those with comorbid PTSD and SAD were compared to those with SAD without PTSD. Nonetheless, such analyses were conducted to provide a basis for comparison and to further highlight the significance of the role of PTSD-SAD comorbidity within the primary analysis which examines the impact of PTSD-SAD comorbidity against that of PTSD alone. Since significant between sex differences have been noted, with higher rates of both SAD (Furmark, 2002) and PTSD (Creamer et al., 2001) documented among females as well as between-sex differences in the types of traumas reported among men and women with PTSD (Breslau, 2002; Creamer et al., 2001; Schnurr et al., 2002; Tolin and Foa, 2002) and SAD (Chartier et al., 2001), between-sex differences in patterns of trauma exposure were explored.

\section{Methods}

\subsection{Participants and procedures}

Data for the current study were gathered from Wave 2 of the National Epidemiologic Survey of Alcohol and Related Conditions (NESARC; $n=34,653$ ). The NESARC is a nationally-representative survey of the United States population, including citizens and noncitizens, aged 18 years and older. Wave 2 of this survey was gathered between 2004 and 2005, and involved an attempt to re-interview the original Wave 1 sample. All interviews were conducted face-to-face within the respondent's household by trained lay United States Census Bureau Field Representatives. Additional information regarding the NESARC sampling design and methodology is provided by Grant et al. (2003).

\subsection{Measures}

The Alcohol Use Disorder and Associated Disabilities Interview Schedule - IV (AUDADIS-IV) was employed to assess the presence of any Axis I disorder. The AUDAIS-IV is a fully structured diagnostic interview, based on criteria set forth in the Diagnostic and Statistical Manual of Mental Disorders, Fourth Edition (DSM-IV; American Psychiatric Association, 2000), appropriate for both clinical and general populations (Grant et al., 2004). The AUDADIS-IV has been reviewed extensively and has been found to be reliable in diagnosing alcohol and psychiatric disorders in the general population, with testretest reliability coefficients in the fair range for the anxiety disorders (Kappa values=0.42-0.64) and the good range for alcohol abuse/ dependence and the mood disorders (Kappa values=0.58-0.70; Grant et al., 2003; Ruan et al., 2008). Within the current study, comorbidity was defined as meeting criteria for both past year PTSD and SAD.

As part of the NESARC Wave 2 interview protocol, all participants were asked whether they had experienced 33 specific and potentially traumatizing events. Seven of these events referred directly to the $9 / 11$ terrorist attack and were excluded from current study because of their highly specific nature; however, more general experiences with acts of terrorism were investigated. Childhood maltreatment was assessed within a separate module. Events relating to abuse and neglect in childhood were included in the current study to provide the most comprehensive assessment of traumatic events possible, and to provide clarification regarding the association between comorbidity and various types of childhood abuse and neglect. Composite childhood maltreatment cluster variables were defined in accordance with prior research and definitions employed in the Adverse Childhood Experiences study (Dong et al., 2003; Dube et al., 2003). For more detailed information regarding construction of childhood abuse and neglect variables please refer to Afifi et al. (2011).

All potentially traumatic events were analysed individually as well as through clustered analysis in order to maintain consistency with prior research (e.g., Raun et al., 2008), and to facilitate comparison of different types of traumatic events. Consistent with prior research on trauma and PTSD (Fetzner et al., 2011), events were grouped into the following six categories: war-related, child maltreatment, assaultive violence, other shocking events, unexpected death, and learned of trauma.

\subsection{Data analysis}

Descriptive statistics were calculated using SPSS (Version 20.0). Due to the complex sampling design used in the development of the 
NESARC, the potential for larger than expected standard errors resulting in inaccurate estimates of statistical significance was anticipated. To address this issue, SUDAAN 10.0.1 statistical software (Research Triangle Institute, 2004) was used for data analysis to adjust the standard error using Taylor Series Linearization, resulting in more accurate estimates of significance. As well, to more accurately represent the United States population, appropriate weights provided by the NESARC were applied.

Pearson's chi-square analyses were first calculated for all sociodemographic variables (age, sex, race, education, marital status, and household income) to identify significant differences between individuals with comorbid PTSD and SAD (PTSD-SAD), those with PTSD without SAD (PTSD-no SAD), and those with SAD without PTSD (SADno PTSD). Multiple logistic regression analyses were then conducted comparing those in the PTSD-SAD group to each of the PTSD-no SAD and SAD-no PTSD groups, with the latter two groups used as reference categories within their respective analyses. For all logistic regression analyses, trauma exposure was used as the dependent variable and diagnosis was used as the independent variable. Regressions were run first after adjusting for all relevant sociodemographic factors and then following adjustment for sociodemographics and all Axis I mood, anxiety (excluding PTSD and SAD), and illicit substance and alcohol use disorders. A conservative $99 \%$ confidence interval was instituted to limit risk of Type 1 error.

The first series of logistic regression analyses assessed the likelihood of exposure to 32 specific traumatic events and six categories of events within the total sample. Insufficient cell size precluded logistic regression analysis for several individual traumas; however, these events were maintained in the clustered trauma categories. Sex stratified analyses were then conducted to examine between sex differences in exposure to trauma clusters and childhood maltreatment variables (e.g., physical abuse, physical neglect). Tables comparing the PTSD-no SAD group to the comorbid PTSD-SAD group are shown. SAD-no PTSD comparisons are discussed in text only and Supplementary tables are available for review online.

\section{Results}

Within the current sample, 1715 individuals (5.0\%; 467 males, 1248 females) met criteria for past-year PTSD, whereas 945 participants (2.7\%; 332 males, 613 females) met criteria for past-year SAD. Of those participants, 217 individuals $(0.6 \%$; 58 males, 159 females) met criteria for past-year diagnoses of both PTSD and SAD. Therefore, among those with past-year PTSD, approximately 13\% met criteria for a comorbid past-year diagnosis of SAD.

Analysis of sociodemographic variables (Table 1) revealed significant differences between groups with regard to sex, marital status, household income, and age.

Table 2 shows significant between group differences in relation to trauma clusters within the total sample. Individuals in the PTSD-SAD group were significantly more likely than those in the SAD-no PTSD group to report having experienced traumas from virtually all categories, with adjusted odds ratio (AOR-1's) ranging from 2.29 (99\% CI: 1.38-3.79) within the unexpected death category to 3.62 (99\% CI: 2.01-6.54) within the other shocking event category. The learned of trauma category was the only association which did not reach statistical significance. Comparisons between the PTSD-SAD and PTSD-no SAD groups revealed more restricted between group differences. Individuals in the PTSD-SAD group were more likely than those in the PTSD-no SAD group to report having experienced traumas from the assaultive violence (AOR-1: 1.93 [99\% CI: 1.11-3.34]), other shocking event (AOR-1: 1.74 [99\% CI: 1.02-2.96]), and childhood maltreatment (AOR-1: 2.27 [99\% CI: 1.29-4.00]) clusters. Following adjustment for sociodemographics and every other Axis I disorder (AOR-2), only the childhood maltreatment cluster remained significant.
Table 1

Sociodemographic factors and comparisons for diagnostic groups.

\begin{tabular}{|c|c|c|c|c|}
\hline $\begin{array}{l}\text { Sociodemographic } \\
\text { Variables }\end{array}$ & $\begin{array}{l}\text { PTSD-no } \\
\text { SAD } \\
(n=1,498) \\
n(\%)\end{array}$ & $\begin{array}{l}\text { SAD-no } \\
\text { PTSD } \\
(n=728) \\
n(\%)\end{array}$ & $\begin{array}{l}\text { PTSD-SAD } \\
(n=217) \\
n(\%)\end{array}$ & $x^{2}$ \\
\hline Sex & & & & $10.46^{* *}$ \\
\hline Men & 409 (30.6) & $274(42.5)$ & $58(28.6)$ & \\
\hline Women & $1089(69.4)$ & $454(57.5)$ & $159(71.4)$ & \\
\hline Education & & & & 2.20 \\
\hline Less than grade 12 & $276(16.1)$ & $95(12.2)$ & $50(20.0)$ & \\
\hline $\begin{array}{l}\text { High school or } \\
\text { equivalent }\end{array}$ & $402(26.8)$ & $225(28.7)$ & $65(31.5)$ & \\
\hline Some college or higher & $820(57.1)$ & $408(59.1)$ & $102(48.5)$ & \\
\hline Marital status & & & & $6.86^{* * *}$ \\
\hline Married/cohabitating & $705(57.2)$ & $304(50.6)$ & $83(46.8)$ & \\
\hline $\begin{array}{l}\text { Separated/divorced/ } \\
\text { widowed }\end{array}$ & $511(27.3)$ & $214(23.6)$ & $86(34.1)$ & \\
\hline Never married & $282(15.6)$ & $210(25.8)$ & $48(19.1)$ & \\
\hline Household income & & & & $3.49^{*}$ \\
\hline$\$ 0-\$ 19,999$ & $480(27.6)$ & $234(26.7)$ & $102(40.1)$ & \\
\hline$\$ 20,000-\$ 34,999$ & $356(22.6)$ & $120(16.8)$ & $35(18.1)$ & \\
\hline$\$ 35,000-\$ 59,999$ & $321(23.2)$ & $193(30.0)$ & $34(16.8)$ & \\
\hline Greater than $\$ 60,000$ & $341(26.6)$ & $181(26.6)$ & $46(25.0)$ & \\
\hline Race & & & & 3.01 \\
\hline White & 831 (69.9) & $461(76.8)$ & $121(67.7)$ & \\
\hline Black & $342(14.0)$ & $124(9.7)$ & $43(11.4)$ & \\
\hline Other & $325(16.0)$ & $143(13.5)$ & $53(20.9)$ & \\
\hline Age & & & & $6.07^{* * *}$ \\
\hline 20-39 years & $497(34.7)$ & $303(45.4)$ & $87(45.0)$ & \\
\hline $40-53$ years & $527(35.4)$ & $255(33.8)$ & $82(35.5)$ & \\
\hline $54+$ years & $474(29.9)$ & $170(20.8)$ & 48 (19.5) & \\
\hline
\end{tabular}

Note. All $n$ s were unweighted. All percentages are weighted. PTSD=posttraumatic stress disorder; $\mathrm{SAD}=$ social anxiety disorder.

${ }^{*} p \leq 0.01$.

$p \leq 0.001$.

Analysis of specific traumatic events (also shown in Table 2) revealed numerous significant differences in patterns of trauma exposure between the PTSD-SAD and SAD-no PTSD groups. Individuals in the PTSD-SAD group were significantly more likely than those in the SAD-no PTSD group to report exposure to trauma. The following three traumas demonstrated the strongest associations within AOR-1: kidnapped/held hostage/POW (5.60; 99\% CI: 1.94-16.14), sexual assault-molestation-rape or unwanted sexual advances (4.10; 99\% CI: 2.37-7.12), and stalked (3.88; 99\% CI: 2.09-7.21). The only associations for which sufficient data was available that did not reach significance in AOR-1 were serious fire/tornado/flood/earthquake/ hurricane, directly experienced a terrorist attack, and someone close experienced a serious/life-threatening illness/accident/injury. The vast majority of significant associations retained significance at AOR2 , with the exception of other very stressful/traumatic event and someone close experienced any other stressful/traumatic experience.

Comparisons between the PTSD-SAD and PTSD-no SAD groups within the assaultive violence category indicated that individuals in the PTSD-SAD group were more likely than those in the PTSD-no SAD group to report experiencing sexual assault, molestation, rape, or unwanted sexual advances (AOR-1: 3.22 [99\% CI: 1.94-5.35]) and kidnapped/held hostage/POW (AOR-1: 2.57 [99\% CI: 1.16-5.68]). Only one trauma within the other shocking event cluster differed significantly between groups, with those in the PTSD-SAD group being significantly more likely than those in the PTSD-no SAD group to report experiencing a serious/life-threatening accident (AOR-1: 1.93 [99\% CI: 1.15-3.25]). Finally, within the childhood maltreatment cluster, individuals in the PTSD-SAD group were significantly more likely than those in the PTSD-no SAD group to report experiencing every category of childhood abuse or neglect, with the strongest AOR1s observed with the following traumas: physical neglect $(1.97 ; 99 \%$ CI: $1.25-3.10)$, sexual abuse (2.31; 99\% CI: 1.47-3.63), and emotional 
Table 2

Individual traumas and trauma clusters for comparison within total sample.

\begin{tabular}{|c|c|c|c|c|}
\hline Traumatic events & $\begin{array}{l}\text { PTSD- } \\
\text { no SAD } \\
n(\%)\end{array}$ & $\begin{array}{l}\text { PTSD- } \\
\text { SAD } n \\
(\%)\end{array}$ & $\begin{array}{l}\text { AOR-1 } \\
99 \% \text { CI }\end{array}$ & $\begin{array}{l}\text { AOR-2 } \\
99 \% \text { CI }\end{array}$ \\
\hline War-related trauma & $\begin{array}{l}179 \\
(13.0)\end{array}$ & $17(7.2)$ & $\begin{array}{l}0.66 \\
(0.26- \\
1.67)\end{array}$ & $\begin{array}{l}0.67 \\
(0.26- \\
1.72)\end{array}$ \\
\hline Military combat & $112(8.4)$ & $8(2.7)$ & $\begin{array}{l}0.44 \\
(0.13- \\
1.50)\end{array}$ & $\begin{array}{l}0.48 \\
(0.14- \\
1.69)\end{array}$ \\
\hline $\begin{array}{l}\text { Peacekeeping/relief work } \\
\text { in a war zone }\end{array}$ & $40(2.7)$ & $4(1.9)$ & N/A & N/A \\
\hline $\begin{array}{l}\text { Unarmed civilian in a } \\
\text { war/revolution/coup }\end{array}$ & $51(3.5)$ & $8(3.8)$ & $\begin{array}{l}1.18 \\
(0.29- \\
4.79)\end{array}$ & $\begin{array}{l}1.08 \\
(0.26- \\
4.46)\end{array}$ \\
\hline Refugee & $33(2.2)$ & $2(1.6)$ & $\mathrm{N} / \mathrm{A}$ & N/A \\
\hline Assaultive violence & $\begin{array}{l}877 \\
(58.1)\end{array}$ & $\begin{array}{l}171 \\
(75.6)\end{array}$ & $\begin{array}{l}1.93 \\
(1.11- \\
3.34)^{*}\end{array}$ & $\begin{array}{l}1.73 \\
(0.98- \\
3.07)\end{array}$ \\
\hline $\begin{array}{l}\text { Physically attacked by } \\
\text { spouse/romantic partner }\end{array}$ & $\begin{array}{l}366 \\
(22.6)\end{array}$ & $84(36.2)$ & $\begin{array}{l}1.69 \\
(0.99- \\
2.87)\end{array}$ & $\begin{array}{l}1.51 \\
(0.88- \\
2.58)\end{array}$ \\
\hline $\begin{array}{l}\text { Physically attacked by } \\
\text { anyone else }\end{array}$ & $\begin{array}{l}257 \\
(18.7)\end{array}$ & $69(28.4)$ & $\begin{array}{l}1.70 \\
(0.96- \\
3.00)\end{array}$ & $\begin{array}{l}1.55 \\
(0.85- \\
2.81)\end{array}$ \\
\hline $\begin{array}{l}\text { Sexual assault, } \\
\text { molestation, rape or } \\
\text { unwanted sexual } \\
\text { advances }\end{array}$ & $\begin{array}{l}410 \\
(26.9)\end{array}$ & $\begin{array}{l}120 \\
(52.5)\end{array}$ & $\begin{array}{l}3.22 \\
(1.94- \\
5.35)^{* *}\end{array}$ & $\begin{array}{l}2.87 \\
(1.72- \\
4.80)^{* *}\end{array}$ \\
\hline Stalked & $\begin{array}{l}305 \\
(20.0)\end{array}$ & $64(29.0)$ & $\begin{array}{l}1.43 \\
(0.88- \\
2.32)\end{array}$ & $\begin{array}{l}1.36 \\
(0.83- \\
2.23)\end{array}$ \\
\hline $\begin{array}{l}\text { Mugged/held up/ } \\
\text { threatened with a weapon }\end{array}$ & $\begin{array}{l}381 \\
(26.7)\end{array}$ & $79(35.5)$ & $\begin{array}{l}1.43 \\
(0.87- \\
2.34)\end{array}$ & $\begin{array}{l}1.28 \\
(0.76- \\
2.17)\end{array}$ \\
\hline $\begin{array}{l}\text { Kidnapped/held hostage/ } \\
\text { POW }\end{array}$ & $69(4.1)$ & $23(11.1)$ & $\begin{array}{l}2.57 \\
(1.16- \\
5.68)^{*}\end{array}$ & $\begin{array}{l}2.78 \\
(1.25- \\
6.18)^{*}\end{array}$ \\
\hline Other shocking event & $\begin{array}{l}1114 \\
(74.8)\end{array}$ & $\begin{array}{l}172 \\
(82.0)\end{array}$ & $\begin{array}{l}1.74 \\
(1.02- \\
2.96)^{*}\end{array}$ & $\begin{array}{l}1.55(0.89, \\
2.07)\end{array}$ \\
\hline $\begin{array}{l}\text { Serious/life-threatening } \\
\text { accident }\end{array}$ & $\begin{array}{l}430 \\
(27.8)\end{array}$ & $88(42.9)$ & $\begin{array}{l}1.93 \\
(1.15- \\
3.25)^{*}\end{array}$ & $\begin{array}{l}1.84 \\
(1.08- \\
3.13)^{*}\end{array}$ \\
\hline $\begin{array}{l}\text { Serious/life-threatening } \\
\text { illness }\end{array}$ & $\begin{array}{l}448 \\
(29.8)\end{array}$ & $79(36.3)$ & $\begin{array}{l}1.53 \\
(0.92- \\
2.56)\end{array}$ & $\begin{array}{l}1.47 \\
(0.87- \\
2.50)\end{array}$ \\
\hline $\begin{array}{l}\text { Serious fire/tornado/ } \\
\text { flood/earthquake/ } \\
\text { hurricane }\end{array}$ & $\begin{array}{l}374 \\
(25.7)\end{array}$ & $54(23.8)$ & $\begin{array}{l}0.93 \\
(0.54- \\
1.59)\end{array}$ & $\begin{array}{l}0.96 \\
(0.56- \\
1.65)\end{array}$ \\
\hline $\begin{array}{l}\text { Injured in a terrorist } \\
\text { attack }\end{array}$ & $4(0.3)$ & $1(0.9)$ & N/A & N/A \\
\hline $\begin{array}{l}\text { Directly experienced a } \\
\text { terrorist attack }\end{array}$ & $39(2.6)$ & $5(2.0)$ & $\begin{array}{l}0.86 \\
(0.20- \\
3.69)\end{array}$ & $\begin{array}{l}0.83 \\
(0.18- \\
3.85)\end{array}$ \\
\hline $\begin{array}{l}\text { Seen someone badly } \\
\text { injured/ killed/ } \\
\text { unexpectedly see a dead } \\
\text { body }\end{array}$ & $\begin{array}{l}623 \\
(42.7)\end{array}$ & $\begin{array}{l}108 \\
(50.6)\end{array}$ & $\begin{array}{l}1.47 \\
(0.91- \\
2.39)\end{array}$ & $\begin{array}{l}1.32 \\
(0.82- \\
2.15)\end{array}$ \\
\hline $\begin{array}{l}\text { Any other very stressful/ } \\
\text { traumatic event }\end{array}$ & $\begin{array}{l}193 \\
(13.2)\end{array}$ & 35 (15.7) & $\begin{array}{l}1.24 \\
(0.65- \\
2.39)\end{array}$ & $\begin{array}{l}1.10 \\
(0.56- \\
2.17)\end{array}$ \\
\hline Unexpected death & $\begin{array}{l}980 \\
(65.8)\end{array}$ & $\begin{array}{l}153 \\
(70.4)\end{array}$ & $\begin{array}{l}1.25 \\
(0.77- \\
2.03)\end{array}$ & $\begin{array}{l}1.08 \\
(0.65- \\
1.80)\end{array}$ \\
\hline $\begin{array}{l}\text { Someone close die in } \\
\text { terrorist attack }\end{array}$ & $38(2.3)$ & $7(2.8)$ & $\begin{array}{l}1.12 \\
(0.33- \\
3.77)\end{array}$ & $\begin{array}{l}1.12 \\
(0.30- \\
4.16)\end{array}$ \\
\hline $\begin{array}{l}\text { Someone close die } \\
\text { unexpectedly }\end{array}$ & $\begin{array}{l}969 \\
(65.2)\end{array}$ & $\begin{array}{l}152 \\
(70.2)\end{array}$ & $\begin{array}{l}1.28 \\
(0.79- \\
2.07)\end{array}$ & $\begin{array}{l}1.11 \\
(0.67- \\
1.83)\end{array}$ \\
\hline Learned of trauma & $\begin{array}{l}1091 \\
(74.3)\end{array}$ & $\begin{array}{l}158 \\
(74.7)\end{array}$ & $\begin{array}{l}1.07 \\
(0.61- \\
1.89)\end{array}$ & $\begin{array}{l}0.93 \\
(0.52- \\
1.67)\end{array}$ \\
\hline $\begin{array}{l}\text { Someone close injured in a } \\
\text { terrorist attack }\end{array}$ & $44(3.2)$ & $6(3.8)$ & $\begin{array}{l}1.18 \\
(0.31- \\
\quad \text { (conti }\end{array}$ & $\begin{array}{l}1.07 \\
(0.28- \\
\text { on next }\end{array}$ \\
\hline
\end{tabular}

Table 2 (continued)

\begin{tabular}{|c|c|c|c|c|}
\hline Traumatic events & $\begin{array}{l}\text { PTSD- } \\
\text { no SAD } \\
n(\%)\end{array}$ & $\begin{array}{l}\text { PTSD- } \\
\text { SAD } n \\
(\%)\end{array}$ & $\begin{array}{l}\text { AOR-1 } \\
99 \% \text { CI }\end{array}$ & $\begin{array}{l}\text { AOR-2 } \\
99 \% \text { CI }\end{array}$ \\
\hline & & & $4.52)$ & 4.06) \\
\hline $\begin{array}{l}\text { Someone close directly } \\
\text { experience a terrorist } \\
\text { attack }\end{array}$ & $118(8.1)$ & $26(12.3)$ & $\begin{array}{l}1.63 \\
(0.73- \\
3.65)\end{array}$ & $\begin{array}{l}1.38 \\
(0.62- \\
3.11)\end{array}$ \\
\hline $\begin{array}{l}\text { Someone close experience } \\
\text { any other serious/ life- } \\
\text { threatening illness/ } \\
\text { accident/ injury }\end{array}$ & $\begin{array}{l}976 \\
(67.1)\end{array}$ & $\begin{array}{l}145 \\
(70.2)\end{array}$ & $\begin{array}{l}1.23 \\
(0.73- \\
2.08)\end{array}$ & $\begin{array}{l}1.12 \\
(0.66- \\
1.90)\end{array}$ \\
\hline $\begin{array}{l}\text { Someone close have any } \\
\text { other stressful/traumatic } \\
\text { experience }\end{array}$ & $\begin{array}{l}414 \\
(27.7)\end{array}$ & $72(31.7)$ & $\begin{array}{l}1.17 \\
(0.75- \\
1.83)\end{array}$ & $\begin{array}{l}1.07 \\
(0.68- \\
1.68)\end{array}$ \\
\hline Childhood maltreatment & $\begin{array}{l}957 \\
(64.1)\end{array}$ & $\begin{array}{l}174 \\
(80.6)\end{array}$ & $\begin{array}{l}2.27 \\
(1.29- \\
4.00)^{* * *}\end{array}$ & $\begin{array}{l}1.92 \\
(1.09- \\
3.39)^{*}\end{array}$ \\
\hline Physical abuse & $\begin{array}{l}526 \\
(34.7)\end{array}$ & $\begin{array}{l}115 \\
(48.9)\end{array}$ & $\begin{array}{l}1.86 \\
(1.20- \\
2.89)^{* *}\end{array}$ & $\begin{array}{l}1.61 \\
(1.01- \\
2.57)^{*}\end{array}$ \\
\hline Emotional abuse & $\begin{array}{l}359 \\
(24.1)\end{array}$ & $93(37.1)$ & $\begin{array}{l}1.89 \\
(1.20- \\
2.98)^{* * *}\end{array}$ & $\begin{array}{l}1.70 \\
(1.08- \\
2.69)^{*}\end{array}$ \\
\hline Sexual abuse & $\begin{array}{l}422 \\
(27.8)\end{array}$ & $\begin{array}{l}108 \\
(47.9)\end{array}$ & $\begin{array}{l}2.31 \\
(1.47- \\
3.63)^{* *}\end{array}$ & $\begin{array}{l}2.17 \\
(1.36- \\
3.44)^{* *}\end{array}$ \\
\hline Physical neglect & $\begin{array}{l}627 \\
(42.6)\end{array}$ & $\begin{array}{l}129 \\
(59.1)\end{array}$ & $\begin{array}{l}1.97 \\
(1.25- \\
3.10)^{* *}\end{array}$ & $\begin{array}{l}1.82 \\
(1.16- \\
2.85)^{* *}\end{array}$ \\
\hline Emotional neglect & $\begin{array}{l}253 \\
(17.6)\end{array}$ & $79(35.2)$ & $\begin{array}{l}2.72 \\
(1.64- \\
4.53)^{* *}\end{array}$ & $\begin{array}{l}2.48 \\
(1.48- \\
4.14)^{* *}\end{array}$ \\
\hline
\end{tabular}

Note. All $n$ s were unweighted. All percentages are weighted. PTSD=posttraumatic stress disorder; $\mathrm{SAD}=$ social anxiety disorder. AOR-1 adjusted for marital status, age, sex, and income. AOR-2 adjusted for marital status, age, sex, income, and every past year Axis I diagnosis except PTSD and SAD. N/A data not available due to insufficient cell size. $p \leq 0.01$.

$p \leq 0.001$.

neglect (20.72; 99\% CI: 1.64-4.53). All associations remained significant at AOR-2.

Sex stratified analyses revealed significant differences between groups in relation to trauma category exposure. Analysis of the PTSD-SAD and SAD-no PTSD groups revealed that males with PTSD-SAD were significantly more likely to report experiencing warrelated trauma (AOR-1: 5.43 [99\% CI: 1.59-18.54]) and other shocking events (AOR-1: 4.91 [99\% CI: 1.03-23.37]). In contrast, women were more likely to report having experienced all trauma categories with the exception of war-related trauma and learned of trauma, with AOR-1 s ranging from 2.21 (99\% CI: 1.22-4.02) for unexpected death to 5.48 (99\% CI: 2.69-11.16) for any assaultive violence. All associations were significant at AOR-2.

No significant differences in trauma category exposure were observed between males in the PTSD-SAD and PTSD-no SAD groups (Table 3 ). In contrast, females in the PTSD-SAD group were more likely than females in the PTSD-no SAD group to report experiencing assaultive violence (AOR-1: 2.90 [99\% CI: 1.45-5.82]) and childhood maltreatment (AOR-1: 3.39 [99\% CI: 1.59-7.23]). All associations remained significant at AOR-2.

Analysis of individual childhood maltreatment variables revealed some of the most striking differences between the sexes (Table 4). Increased rates of childhood maltreatment among those in the PTSDSAD group, relative to the SAD-no PTSD and PTSD-no SAD groups, were found among women only. Significant associations were found across all abuse and neglect categories and all levels of adjustment. For the PTSD-SAD and SAD-no PTSD comparisons, the strongest AOR-1s were observed among the following traumas: physical neglect (2.69; 99\% CI: 1.58-4.52), physical abuse (2.74; 99\% CI 1.49-5.03), and sexual abuse (3.43; 99\% CI 1.83-6.41). A similar pattern was observed 
Table 3

Trauma clusters for comparison within sex stratified samples.

\begin{tabular}{|c|c|c|c|c|}
\hline Traumatic events & $\begin{array}{l}\text { PTSD-no } \\
\text { SAD } n(\%)\end{array}$ & $\begin{array}{l}\text { PTSD- } \\
\text { SAD } n(\%)\end{array}$ & $\begin{array}{l}\text { AOR-1 99\% } \\
\text { CI }\end{array}$ & $\begin{array}{l}\text { AOR-2 99\% } \\
\text { CI }\end{array}$ \\
\hline \multicolumn{5}{|l|}{ Males } \\
\hline $\begin{array}{l}\text { War-related } \\
\text { trauma }\end{array}$ & $130(31.3)$ & $13(17.2)$ & $\begin{array}{l}0.65(0.25- \\
1.71)\end{array}$ & $\begin{array}{l}0.65(0.24- \\
1.76)\end{array}$ \\
\hline Assaultive violence & $220(54.3)$ & $38(57.4)$ & $\begin{array}{l}1.03(0.36- \\
2.94)\end{array}$ & $\begin{array}{l}0.91(0.29- \\
2.80)\end{array}$ \\
\hline $\begin{array}{l}\text { Other shocking } \\
\text { event }\end{array}$ & $350(85.5)$ & $51(89.4)$ & $\begin{array}{l}1.80(0.39- \\
8.28)\end{array}$ & $\begin{array}{l}1.69(0.36- \\
7.95)\end{array}$ \\
\hline Unexpected death & $274(67.8)$ & $42(71.9)$ & $\begin{array}{l}1.34(0.50- \\
3.59)\end{array}$ & $\begin{array}{l}1.21(0.44- \\
3.33)\end{array}$ \\
\hline Learned of trauma & $290(70.9)$ & $44(73.6)$ & $\begin{array}{l}1.19(0.43- \\
3.27)\end{array}$ & $\begin{array}{l}0.86(0.30- \\
2.41)\end{array}$ \\
\hline $\begin{array}{l}\text { Childhood } \\
\text { maltreatment }\end{array}$ & $266(66.7)$ & $41(67.1)$ & $\begin{array}{l}1.13(0.39- \\
3.28)\end{array}$ & $\begin{array}{l}0.92(0.30- \\
2.85)\end{array}$ \\
\hline \multicolumn{5}{|l|}{ Females } \\
\hline $\begin{array}{l}\text { War-related } \\
\text { trauma }\end{array}$ & 49 (4.9) & $4(3.3)$ & $\begin{array}{l}0.69(0.12- \\
4.03)\end{array}$ & $\begin{array}{l}0.72(0.12- \\
4.38)\end{array}$ \\
\hline Assaultive violence & 657 (59.9) & $133(82.9)$ & $\begin{array}{l}2.90 \\
(1.45- \\
5.82)^{* * *}\end{array}$ & $\begin{array}{l}2.62 \\
(1.29- \\
5.33)^{* * *}\end{array}$ \\
\hline $\begin{array}{l}\text { Other shocking } \\
\text { event }\end{array}$ & $764(70.1)$ & $121(79.1)$ & $\begin{array}{l}1.76(0.98- \\
3.17)\end{array}$ & $\begin{array}{l}1.55(0.83- \\
2.89)\end{array}$ \\
\hline Unexpected death & 706 (64.9) & $111(69.8)$ & $\begin{array}{l}1.26(0.71- \\
2.22)\end{array}$ & $\begin{array}{l}1.06(0.59- \\
1.90)\end{array}$ \\
\hline Learned of trauma & $801(75.7)$ & $114(75.1)$ & $\begin{array}{l}1.05(0.54- \\
2.03)\end{array}$ & $\begin{array}{l}1.00(0.49- \\
2.01)\end{array}$ \\
\hline $\begin{array}{l}\text { Childhood } \\
\text { maltreatment }\end{array}$ & $691(63.0)$ & $133(85.8)$ & $\begin{array}{l}3.39 \\
(1.59- \\
7.23)^{* * *}\end{array}$ & $\begin{array}{l}2.92 \\
(1.38- \\
6.19)^{* * *}\end{array}$ \\
\hline
\end{tabular}

Note. All $n$ s were unweighted. All percentages are weighted. PTSD=posttraumatic stress disorder; $\mathrm{SAD}=$ social anxiety disorder.

AOR-1 adjusted for marital status, age, and income. AOR-2 adjusted for marital status, age, income, and every past year Axis I diagnosis except PTSD and SAD. ${ }^{*} p \leq 0.01$.

$p \leq 0.001$.

within the PTSD-SAD and PTSD-no SAD comparisons, where the strongest AOR-1s were observed among the following traumas: physical neglect $(2.45 ; 99 \%$ CI: $1.50-3.99)$, emotional neglect $(2.74 ; 99 \%$ CI: $1.53-4.88)$, and sexual abuse (3.00; 99\% CI: 1.76-5.12). All sex stratified associations remained significant at AOR-2.

\section{Discussion}

The experience of trauma is central to the development of PTSD, and emerging evidence suggests that such experiences may also precipitate the development of SAD. It is essential that the impact of trauma be further explored to provide insight into factors that may contribute to the high rates of comorbidity observed between these disorders. The current study sought to clarify and extend previous research through examination of comorbid PTSD and SAD, compared to PTSD without SAD and SAD without PTSD, in relation to a broad range of specific traumas and trauma clusters within a nationallyrepresentative sample. Additionally, the present study sought to clarify the impact of between sex differences on the nature of such associations.

Results of the current study revealed a unique pattern of trauma exposure among those with comorbid PTSD-SAD relative to those with PTSD-no SAD and SAD-no PTSD. Even after adjusting for the presence of other comorbid conditions, those in the PTSD-SAD group were more likely than those in either the PTSD-no SAD or SAD-no PTSD groups to report having experienced assaultive violence, other shocking events, or childhood maltreatment. Additionally, substantial between-sex differences suggested the influence childhood maltreatment on PTSD and SAD comorbidity may be particularly robust among females.

As anticipated, individuals in the PTSD-SAD group were more likely than those in the SAD-no PTSD group to report experiencing the
Table 4

Childhood maltreatment variables for comparison within sex stratified samples.

\begin{tabular}{|c|c|c|c|c|}
\hline $\begin{array}{l}\text { Childhood } \\
\text { maltreatment }\end{array}$ & $\begin{array}{l}\text { PTSD-no } \\
\text { SAD } n(\%)\end{array}$ & $\begin{array}{l}\text { PTSD- } \\
\text { SAD } n(\%)\end{array}$ & $\begin{array}{l}\text { AOR-1 99\% } \\
\text { CI }\end{array}$ & $\begin{array}{l}\text { AOR-2 99\% } \\
\text { CI }\end{array}$ \\
\hline \multicolumn{5}{|l|}{ Males } \\
\hline Physical abuse & $151(37.1)$ & $27(42.3)$ & $\begin{array}{l}1.43(0.62- \\
3.32)\end{array}$ & $\begin{array}{l}1.18(0.48- \\
2.91)\end{array}$ \\
\hline Emotional abuse & $95(22.3)$ & $18(23.4)$ & $\begin{array}{l}1.14(0.45- \\
2.89)\end{array}$ & $\begin{array}{l}0.94(0.36- \\
2.48)\end{array}$ \\
\hline Sexual abuse & $61(14.5)$ & $11(12.6)$ & $\begin{array}{l}0.84(0.20- \\
3.49)\end{array}$ & $\begin{array}{l}0.76(0.18- \\
3.29)\end{array}$ \\
\hline Physical neglect & $198(50.2)$ & $32(54.8)$ & $\begin{array}{l}1.15(0.43- \\
3.09)\end{array}$ & $\begin{array}{l}1.06(0.37- \\
2.98)\end{array}$ \\
\hline Emotional neglect & $51(13.7)$ & $15(27.9)$ & $\begin{array}{l}2.88(0.97- \\
8.59)\end{array}$ & $\begin{array}{l}2.40(0.82- \\
6.97)\end{array}$ \\
\hline \multicolumn{5}{|l|}{ Females } \\
\hline Physical abuse & 375 (33.6) & $88(51.5)$ & $\begin{array}{l}2.10 \\
(1.21- \\
3.62)^{* *}\end{array}$ & $\begin{array}{l}1.86 \\
(1.05- \\
3.28)^{*}\end{array}$ \\
\hline Emotional abuse & $264(24.8)$ & $75(42.6)$ & $\begin{array}{l}2.27 \\
(1.28- \\
4.01)^{* * *}\end{array}$ & $\begin{array}{l}2.13 \\
(1.20- \\
3.77)^{* * *}\end{array}$ \\
\hline Sexual abuse & $361(33.7)$ & $97(61.4)$ & $\begin{array}{l}3.00 \\
(1.76- \\
5.12)^{* *}\end{array}$ & $\begin{array}{l}2.85 \\
(1.66- \\
4.90)^{* *}\end{array}$ \\
\hline Physical neglect & $429(39.2)$ & $97(60.9)$ & $\begin{array}{l}2.45 \\
(1.50- \\
3.99)^{* *}\end{array}$ & $\begin{array}{l}2.28 \\
(1.40- \\
3.70)^{* * *}\end{array}$ \\
\hline Emotional neglect & $202(19.2)$ & $64(38.1)$ & $\begin{array}{l}2.74 \\
(1.53- \\
4.88)^{* *}\end{array}$ & $\begin{array}{l}2.59 \\
(1.43- \\
4.70)^{* *}\end{array}$ \\
\hline
\end{tabular}

Note. All $n$ s were unweighted. All percentages are weighted. PTSD=posttraumatic stress disorder; $\mathrm{SAD}=$ social anxiety disorder.

AOR-1 adjusted for marital status, age, and income. AOR-2 adjusted for marital status, age, income, and every past year Axis I diagnosis except PTSD and SAD.

${ }^{*} p \leq 0.01$.

$p \leq 0.001$.

majority of individual traumas and trauma categories investigated, underscoring the clear relevance of trauma exposure to the emergence of PTSD. Specific hypotheses regarding the association between interpersonal and assaultive factors in relation to PTSD-SAD comorbidity were also largely supported. Within the total sample, individuals in the PTSD-SAD group were more likely than those in either the PTSD-no SAD or SAD-no PTSD groups to report experiencing traumas involving physical violence, sexual assault, and all types of childhood abuse or neglect. Comparison between the PTSD-SAD and PTSD-no SAD groups revealed several individual traumas within the assaultive violence category which approached significance; however, results may have been restricted due to the use of conservative significance estimates.

The hypothesized relationship between childhood abuse and neglect and PTSD-SAD comorbidity was strongly supported. Indeed, childhood physical abuse, emotional abuse, sexual abuse, physical neglect, and emotional neglect were all more common among those with comorbid PTSD-SAD compared to those with PTSD-no SAD or SAD-no PTSD. Such results extend prior research demonstrating associations with SAD and PTSD individually (Acarturk et al., 2009; Bandelow et al., 2004; Creamer et al., 2001; Kuo et al., 2011) to demonstrate strong associations between all forms of childhood abuse and neglect and PTSD-SAD comorbidity.

Observed associations between PTSD-SAD comorbidity and sexual assault and childhood abuse and neglect, may be due, in part, to guilt and shame regarding such traumas. Beyond previously discussed findings (Magee, 1999; Orsillo, et al., 1996), Hofmann, 2007 and Foa and Kozak's (1986) Emotional Processing Model of PTSD both offer support for this hypothesis. Specifically, Hofmann's model incorporates shame and guilt in the form of rumination among individuals with $\mathrm{SAD}$, while Foa's Emotional Processing Model integrates such responses within the context of negative appraisals of one's response to 
trauma, and in the eyes of others, among individuals with PTSD. Such theoretical formulations may be particularly relevant for sexual assault due to self-blame and stigmatization which frequently accompanies sexual victimization (Pietrzak et al., 2011). Among persons who were sexually abused during childhood, condemnation by the perpetrator and threats to keep silent may further contribute to shame and guilt, resulting in perceptions of stigma as it reinforces a child's sense of being different (Feiring and Taska, 2005). Further research is needed in this area.

Transdiagnostic approaches to the treatment of emotional disorders provide further context within which to understand the nature of PTSD-SAD comorbidity following exposure to trauma such as sexual violence. Within a transdiagnostic framework, comorbidity is viewed as variation in the expression of shared factors (e.g., negative affectivity, locus of control, emotion dysregulation) which contribute to the development and maintenance of emotional disorders (Allen et al., 2008; McEvoy et al., 2008). Transdiagnostic treatments such as the Unified Protocol described by Allen et al., attempt to modify these shared factors by addressing core features underlying emotional disorders in general (e.g., cognitive misappraisals, emotional avoidance, emotion driven behaviours [reactive behaviours associated with each emotion]). Among individuals who have experienced sexual assault as a child or adult, the belief that one is "ruined" by the experience may lead to feelings of social inadequacy within intimate relationships and an overestimation of the potentially negative social outcomes of engaging with potential partners, resulting in symptoms of $\mathrm{SAD}$. However, if the same person were also to overestimate the likelihood of further sexual victimization, associating environmental stimuli with memories of fear and terror, symptoms of PTSD such as hypervigilance, increased startle response, and flashbacks may occur. As such the shared experience of trauma may contribute to the development of psychopathology within the context of other factors, such as impaired emotion regulation, with disorder specific symptoms emerging in response to the type of cognitive, emotional, and behavioural changes experienced.

Changes to the DSM-5 highlight the potential for an individual to develop PTSD upon learning of traumatic events which have directly impacted a close family member or loved one as well as the potential for indirect exposure to aversive details of a traumatic event to precipitate the emergence of PTSD. Interestingly, only one event within the learned of trauma category emerged as significant in the current analyses (someone close to you had any other stressful/traumatic experience); however, this trauma was significant at AOR-1 for the SAD-no PTSD comparisons only. The lack of association between learned of traumas and comorbidity in comparison to the presence of $\mathrm{SAD}$ alone after adjusting for other comorbid disorders highlights the findings of the literature surrounding PTSD, which suggests that traumas that are directly experienced demonstrate some of the strongest associations with PTSD.

The impact of sex on the relationship between trauma type and PTSD-SAD comorbidity was a striking result of the current study, and a novel contribution to the literature. Specifically, cluster level analysis revealed distinct patterns of trauma exposure among males and females diagnosed with comorbid PTSD-SAD compared to those with PTSD-no SAD or SAD-no PTSD.

Examination of individual childhood abuse and neglect variables demonstrated a clear and robust relationship with sex. Females with comorbid PTSD and SAD were significantly more likely that those with either disorder alone to report a history of childhood abuse or neglect. No such associations were observed among males. Sex-related differences in the prevalence of both PTSD and SAD are well documented in the literature (Creamer et al., 2001; Furmark, 2002); however, the current study is the first to demonstrate between-sex differences in the relationship between trauma and comorbid PTSD and SAD. The discrepancies observed between males and females within the current study underscores the need for further research into the causes and correlates of comorbid PTSD and SAD among women. Indeed, the majority of research in this area has not included an examination of between-sex differences, a significant limitation given that the results of the current study suggest that the link between trauma type and PTSD-SAD comorbidity may manifest quite differently between the sexes. Such matters are particularly important in studies utilizing military populations, which generally include a disproportionately low number of female participants, potentially obscuring important differences between groups.

There are several limitations to the current study that can be regarded as areas for future inquiry. First, restrictions due to sample size precluded analysis of several individual traumas within the total sample and constrained sex stratified analyses to an examination of cluster level traumas only. Given the significance of the between sex differences observed with respect to childhood maltreatment variables, it is recommended that future studies utilize a balanced sample of males and females to allow for a detailed analysis of between sex differences. Second, while diagnoses were made using past-year criteria, it is was not possible to determine participant age at the time of trauma exposure on onset of diagnosis. Third, although still likely representative of the diagnostic categories studied herein, the current study utilized the now dated DSM-IV diagnostic criteria for identification of PTSD and SAD. Finally, diagnoses were made via a reliable structured interview conducted by professional interviewers; however, these diagnoses may not be as accurate or reliable as those made by trained clinicians.

To the best of our knowledge, the current study represents the most in-depth epidemiological evaluation of the relationship between traumatic life events and the presence of PTSD-SAD comorbidity to date, demonstrating significant differences in trauma histories among those with comorbid PTSD and SAD in comparison to those with PTSD or $\mathrm{SAD}$ alone. In addition, between sex differences were investigated and yielded results that are applicable to the population as a whole. Results demonstrated a robust association between childhood maltreatment and PTSD-SAD comorbidity which was present among females only. This unique pattern was previously unrecognized within the literature and warrants further investigation. The majority of individuals with SAD who present for treatment do so to treat a comorbid condition, and not SAD specifically; as such, clinicians treating individuals with PTSD should be aware of the relationship between trauma history and the presence of PTSD-SAD comorbidity to facilitate case conceptualization and inform clinical practice.

\section{Acknowledgements}

Katherine A. McMillan was supported by CIHR Canada Graduate Scholarship Doctoral Research Award (FRN: 110589). Gordon J. G. Asmundson was supported by a President's Chair for Academic Excellence in Adult Mental Health Research. Conflicts of interest: none. The authors would also like to thank Matthew G. Fetzner for his assistance proof-reading this manuscript.

\section{Appendix A. Supplementary material}

Supplementary data associated with this article can be found in the online version at http://dx.doi.org/10.1016/j.psychres.2016.10.036.

\section{References}

Acarturk, C., Smit, F., de Graaf, R., van Straten, A., ten Have, M., Cuijpers, P., 2009. Incidence of social phobia and identification of its risk indicators: a model for prevention. Acta Psychiatr. Scand. 119, 62-70. http://dx.doi.org/10.1111/j.1600$0447.2008 .01275 \mathrm{x}$.

Afifi, T.O., McMillan, K.A., Asmundson, G.J.G., Pietrzak, R.H., Sareen, J., 2011. An examination of the relation between conduct disorder, childhood and adulthood traumatic events, and posttraumatic stress disorder in a nationally representative sample. J. Psychiatr. Res 45, 1564-1572. http://dx.doi.org/10.1016/ 
j.jpsychires.2011.08.005.

American Psychiatric Association, 2000. Diagnostic and Statistical Manual of Mental Disorders, 4th ed., text revision. Author, Washington, D.C.

American Psychiatric Association, 2013. Diagnostic and Statistical Manual of Mental Disorders, 5th ed. Author, Washington, D.C.

Bandelow, B., Torente, A.C., Wedekind, D., Broocks, A., Hajak, G., Ruther, E., 2004 Early traumatic life events, parental rearing styles, family history of mental disorders, and birth risk factors in patients with social anxiety disorder. Eur. Arch Psychiatry Clin. Neurosci. 254, 379-405. http://dx.doi.org/10.1007/s00406-0040521-2.

Allen, L.B., McHugh, R.K., Barlow, D.H., 2008. Emotional disorders: a unified protocol. In: Barlow, D.H. (Ed.), Clinical Handbook of Psychological Disorders: A Step-ByStep Treatment Manual4th ed.. Guilford Press, New York, 216-249.

Breslau, N., 2002. Epidemiologic studies of trauma, posttraumatic stress disorder, and other psychiatric disorders. Can. J. Psychiatry 47 (10), 923-929.

Collimore, K.C., Asmundson, G.J.G., Taylor, S., Jang, K.L., 2009. Socially related fears following exposure to trauma: environmental and genetic influences. J. Anxiety Disord. 23, 240-246. http://dx.doi.org/10.1016/j.janxdis.2008.07.006.

Collimore, K.C., Carleton, R.N., Hofmann, S.G., Asmundson, G.J.G., 2010. Posttraumatic stress and social anxiety: the interaction of traumatic events and interpersonal fears. Depress. Anxiety 27, 1017-1026. http://dx.doi.org/10.1002/da.20728.

Chartier, M.J., Walker, J.R., Stein, M.B., 2001. Social phobia and potential childhood risk factors in a community sample. Psychol. Med. 31, 307-315. http://dx.doi.org/ 10.1017/S0033291701003348.

Creamer, M., Burgess, P., McFarlane, A.C., 2001. Post-traumatic stress disorder: findings from the Australian National Survey of Mental Health and Well-being. Psychol. Med. 31, 1237-1247. http://dx.doi.org/10.1017/S0033291701004287.

Dong, M., Anda, R.F., Dube, S.R., Giles, W.H., Felitti, V.J., 2003. The relationship of exposure to childhood sexual abuse to other forms of abuse, neglect, and household dysfunction during childhood. Child Abuse Negl. 27, 625-639. http://dx.doi.org/ 10.1016/S0145-2134(03)00105-4.

Dube, S.R., Felitti, V.J., Dong, M., Chapman, D.P., Giles, W.H., Anda, R.F., 2003. Childhood abuse, neglect, and household dysfunction and the risk of illicit drug use: the adverse childhood experiences study. Paediatrics 111, 564-572. http:// dx.doi.org/10.1542/peds.111.3.564.

Feiring, D., Taska, L.S., 2005. The persistence of shame following sexual abuse: a longitudinal look at risk and recovery. Child Maltreat. 10 (4), 337-349. http:// dx.doi.org/10.1177/1077559505276686.

Fetzner, M.G., McMillan, K.A., Sareen, J., Asmundson, G.J.G., 2011. What is the association between traumatic life events and alcohol abuse/dependence in people with and without PTSD? Findings from a nationally representative sample. Depress. Anxiety 28, 632-638. http://dx.doi.org/10.1002/da.20852.

Foa, E.B., Kozak, M.J., 1986. Emotional processing of fear: exposure to corrective information. Psychol. Bull. 99 (1), 20-35.

Furmark, T., 2002. Social phobia: overview of community surveys. Acta Psychiatr. Scand. 105, 84-93. http://dx.doi.org/10.1034/j.1600-0447.2002.1r103.x.

Golding, J.M., 1999. Intimate partner violence as a risk factor for mental disorders: a meta-analysis. J. Fam. Violence 14, 99-132. http://dx.doi.org/10.1023/ A: 1022079418229

Grant, B.F., Kaplan, K., Shepard, J., Moore, T.C., 2003. Source and Accuracy Statement for Wave 1 of the National Epidemiologic Survey on Alcohol and Related Conditions (NESARC). National Institute on Alcohol abuse and Alcoholism, Bethesda, MD.
Grant, B.F., Stinson, F.S., Dawson, D.A., Chou, S.P., Dufour, M.C., Compton, W., et al., 2004. Prevalence and Co-occurrence of substance use disorders and independent mood and anxiety disorders: results from the national epidemiologic survey on alcohol and related conditions. Arch. Gen. Psychiatry 61, 807-816.

Hofmann, S.G., 2007. Cognitive factors that maintain social anxiety disorders: a comprehensive model and its treatment applications. Cogn. Behav. Ther. 36 193-209. http://dx.doi.org/10.1080/16506070701421313.

Howgego, I.M., Owen, C., Meldrum, L., Yellowles, P., Dark, F., Parslow, R., 2005. Posttraumatic stress disorder: an exploratory study examining rates of trauma and PTSD and its effect on client outcomes in community mental health. BMC Psychiatry 5, 1-17. http://dx.doi.org/10.1186/1471-244X-5-21.

Kashdan, T.B., Frueh, B.C., Knapp, R.G., Hebert, R., Magruder, K.M., 2006. Social anxiety disorder in veteran's affairs primary care clinics. Behav. Res. Ther. 44, 233-247. http://dx.doi.org/10.1016/j.brat.2005.02.002.

Kim, B.N., Kim, J.W., Kim, H.W., Shin, M.S., Cho, S.C., Choi, N.H., et al., 2009. A 6month follow-up study of posttraumatic stress and anxiety/depressive symptoms in Korean children after direct or indirect exposure to a single incident of trauma. J. Clin. Psychiatry 70 (8), 1148-1154.

Kuo, J.R., Goldin, P.R., Werner, K., Heimberg, R.G., Gross, J.J., 2011. Childhood trauma and current psychological functioning in adults with social anxiety disorder. J. Anxiety Disord. 25 (4), 467-473. http://dx.doi.org/10.1016/j.janxdis.2010.11.011.

Magee, W.J., 1999. Effects of negative life experiences on phobia onset. Soc. Psychiatry Psychiatr. Epidemiol. 34, 343-351. http://dx.doi.org/10.1007/s001270050154.

McEvoy, P.M., Nathan, P., Norton, P.J., 2008. Efficacy of transdiagnostic treatments: a review of published outcome studies and future research directions. J. Cogn. Psychother.: Int. Q. 23 (1), 20-33. http://dx.doi.org/10.1891/0889-8391.23.1.20.

Orsillo, S.M., Heimberg, R.G., Juster, H.R., Garrett, J., 1996. Social phobia and PTSD in Vietnam veterans. J. Trauma Stress 9, 235-251. http://dx.doi.org/10.1007/ BF02110658.

Ost, L.G., 1987. Age of onset of different phobias. J. Abnorm. Psychol. 96 (3), 223-229.

Ost, L.G., Hugdahl, K., 1981. Acquisition of phobias and anxiety response patterns in clinical patients. Behav. Res Ther. 19 (5), 439-447.

Pietrzak, R.H., Goldstein, R.B., Southwick, S.M., Grant, B.F., 2011. Prevalence and axis I comorbidity of full and partial posttraumatic stress disorder in the United States: results from Wave 2 of the National Epidemiologic Survey on Alcohol and Related Conditions. J. Anxiety Disord. 25, 456-465, doi: 10.1016éj.janxdis.2010.11.010.

Raun, W.J., Goldstein, R.B., Chou, S.P., Smith, S.M., Saha, T.D., Pickring, R.P., et al., 2008. The alcohol use disorder and associated disabilities interview schedule-IV (AUDADIS-IV): reliability of new psychiatric diagnostic modules and risk factors in a general population sample. Drug Alcohol Depend. 92, 27-36, doi: 10.1016éj.drugalcdep.2007.06.001.

Schnurr, P.P., Friedman, M.J., Bernardy, N.C., 2002. Research on posttraumatic stress disorder: epidemiology, pathophysiology, and assessment. Psychother. Pract. 58, 877-889. http://dx.doi.org/10.1002/jclp.10064.

Stemberger, R.T., Turner, S.M., Beidel, D.C., Calhoun, K.S., 1995. Social phobia: an analysis of possible developmental factors. J. Abnorm. Psychol. 104 (3), 526-531.

Tolin, D.F., Foa, E.B., 2002. Gender and PTSD: a cognitive model. In: Kimerling, R., Ouimette, P., Wolfe, J. (Eds.), Gender and PTSD. Guilford Press, New York, 76-97.

Zayfert, C., DeViva, J.C., Hofmann, S.G., 2005. Comorbid PTSD and social phobia in a treatment-seeking population: an exploratory study. J. Nerv. Ment. Dis. 193, 93-101 . http://dx.doi.org/10.1097/01.nmd.0000152795.47479.d9. 